\title{
Investigation of the Presence and Mechanisms of Action Potential Alternans in Hypertrophic Cardiomyopathy
}

\author{
Aurore Lyon, Ana Mincholé, Elisa Passini, Blanca Rodriguez \\ Department of Computer Science, Oxford, UK
}

\begin{abstract}
Hypertrophic cardiomyopathy (HCM) is a common cardiac genetic disorder. It is a leading cause of sudden cardiac death in young adults, yet most patients remain asymptomatic. $T$ wave alternans (TWA) have been proposed as a potential tool for HCM risk stratification [1] [2] [3] but the mechanisms at stake remain unclear [4] [5]. HCM has been associated with a dysregulation of $\mathrm{Ca}^{2+}$ cycling and an elevation in intracellular calcium concentration [6] that may affect the cardiac action potential (AP). Our goal is to investigate whether these electrophysiological abnormalities may be a mechanism responsible for the presence of TWA in HCM. We use a population of 9,118 control and HCM models defined in [7] and measure alternans in $\mathrm{AP}$ and $\mathrm{Ca}^{2+}$ transient biomarkers for pacing cycle lengths from $250 \mathrm{~ms}$ to 700ms. HCM cardiomyocytes show a distinct AP phenotype with longer $\mathrm{AP}$ and $\mathrm{Ca}^{2+}$ duration $(486 \pm 84 \mathrm{~ms}$

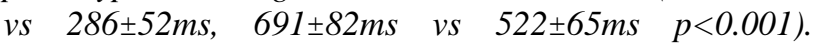
However, there is no significant difference in alternans propensity in HCM compared to controls, except for AP amplitude at low pacing lengths (300ms: $13.4 \%$ HCM models, $2.6 \%$ control models, $p<0.001)$. HCM amplitude and duration alternans also presented larger magnitude than Controls. These findings suggest that TWA in HCM may be related to whole-organ tissue heterogeneities rather than an alteration of the cellular substrate.
\end{abstract}

\section{Introduction}

Hypertrophic cardiomyopathy (HCM) is a cardiac genetic disease and a major cause of sudden cardiac death in young adults. However, most patients remain asymptomatic, and identifying the patients at risk is therefore critical to provide them with appropriate treatment. Several studies [1] [2] [3] have suggested T wave alternans (TWA) as a potential tool to identify these high risk patients, but the mechanisms behind TWA are still unclear [4] [5]. HCM hearts suffer from electrophysiological abnormalities affecting the repolarisation phase. They show an increase of L-type calcium, an increase of Late Sodium current and a reduction of potassium currents [6] [7]. These abnormalities in the calcium cycling may lead to action potential (AP) alternans [8] [9]. Our goal is to investigate whether these electrophysiological abnormalities may be a mechanism responsible for the presence of TWA in HCM. We used a population of model approach described in [7] allowing us to account for variability in HCM and control populations. We analysed two consecutive heart beats at steady state, recorded AP for both populations and compared the presence of alternans in AP biomarkers at different pacing lengths.

\section{Methods}

\subsection{HCM and control populations}

In order to account for variability in HCM and control populations, we used a population of models approach, described in [7]. The baseline model used is the endocardial O'Hara-Rudy model [10] in which 11 parameters are varied to build the control population $(n=9,118)$, in agreement with experimental data described in [6]. The HCM population is then derived from controls by introducing changes in the ionic currents, recreating HCM electrophysiology. As described in [7], INaL was increased by $165 \%$, and ICaL by $40 \%$. Ito was decreased by $70 \%$ and $\mathrm{IK}_{1}$ by $30 \%$. Fast and slow time constants of voltage and $\mathrm{Ca}^{2+}$ dependent $\mathrm{ICaL}$ inactivation were increased by $35 \%$ and $20 \%$ respectively. In addition, IKr and IKs were decreased by $45 \%$, the SERCA pump by $25 \%$, RyRs release by $20 \%$ and $\mathrm{Na}^{+} / \mathrm{Ca}^{2+}$ exchanger was increased by $30 \%$. Troponin for $\mathrm{Ca}^{2+}$ was reduced by $50 \%, \mathrm{Na}^{+} / \mathrm{K}^{+}$pump was reduced by $30 \%$ and the background $\mathrm{Na}^{+}$current was increased by $165 \%$ similarly as INaL.

\subsection{Action potential simulations}

The models were paced until steady state $(500 \mathrm{~ms})$ to ensure stable ionic concentrations of $\mathrm{Na}^{+}$and $\mathrm{K}^{+}$, at the following pacing cycle lengths: $700 \mathrm{~ms}, 600 \mathrm{~ms}, 500 \mathrm{~ms}$, $450 \mathrm{~ms}, 400 \mathrm{~ms}, 350 \mathrm{~ms}, 300 \mathrm{~ms}$ and $250 \mathrm{~ms}$, in order to 
investigate the apparition of alternans at different frequencies. We recorded AP and calcium transient $(\mathrm{CaT})$ traces for two consecutive beats at steady state for the different pacing cycle lengths. Simulations were performed using Chaste, an open source cardiac simulation software [11] and the analysis of AP and CaT traces was performed using custom Matlab software.

\subsection{Biomarkers computation}

In order to compute AP and CaT biomarkers, we discarded the models that failed to repolarize during the simulation, for each cycle length. The following biomarkers were computed:

- $\quad$ Action potential amplitude (APA): computed as the absolute value of the difference between the maximum of the AP and the resting membrane potential

- Action potential duration (APD): computed between the upstroke of the AP and the time of 90\% (APD90) of repolarization.

- Calcium transient (CaT) duration: computed as the maximum of the CaT trace between the upstroke of the CaT trace and the time of $90 \%$ (CaT90) of repolarization.

- $\quad$ CaT amplitude (CaTA): computed as the

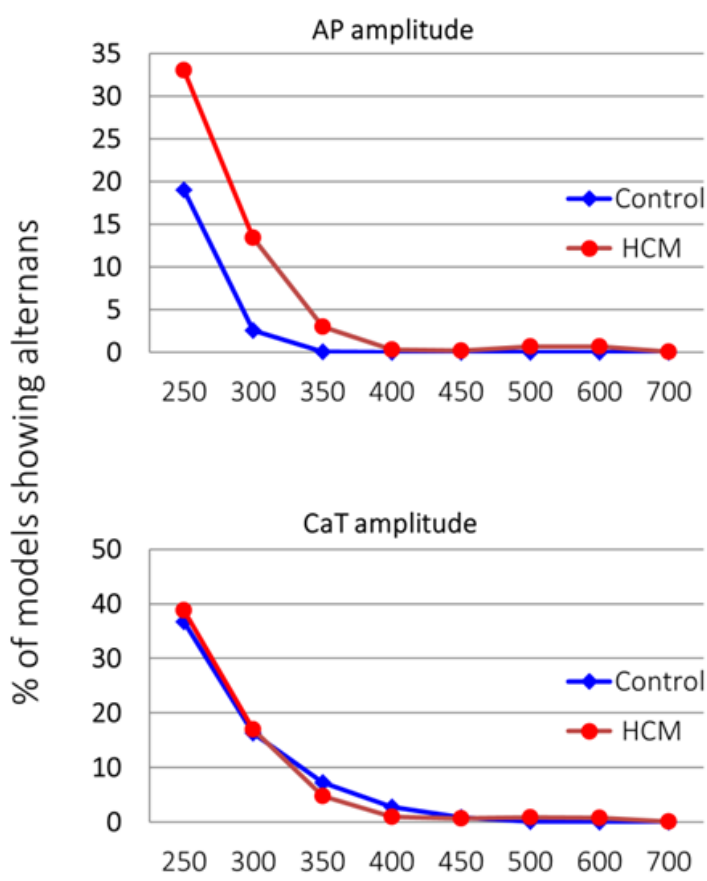

absolute value of the difference between the maximum of the CaT trace and the resting membrane potential

The model was considered to present alternans if the difference in amplitude (resp. duration) between the two consecutive beats was more than $1 \%$ of the magnitude of the amplitude of the first beat (resp. $5 \mathrm{~ms}$ ). In addition to the number of models exhibiting alternans, we computed the magnitude of these alternans for both groups, measured as the absolute difference between the two consecutive beats.

\section{Results}

\subsection{HCM models phenotype}

In agreement with HCM experimental phenotype [7], the HCM models showed a longer APD compared to controls, with greater variability $(486 \pm 84 \mathrm{~ms}$ vs $286 \pm 52 \mathrm{~ms}, \mathrm{p}<0.001)$, as well as a longer CaT duration, with greater variability $(690 \pm 81 \mathrm{~ms}$ vs $522 \pm 65 \mathrm{~ms}$, $\mathrm{p}<0.001)$.
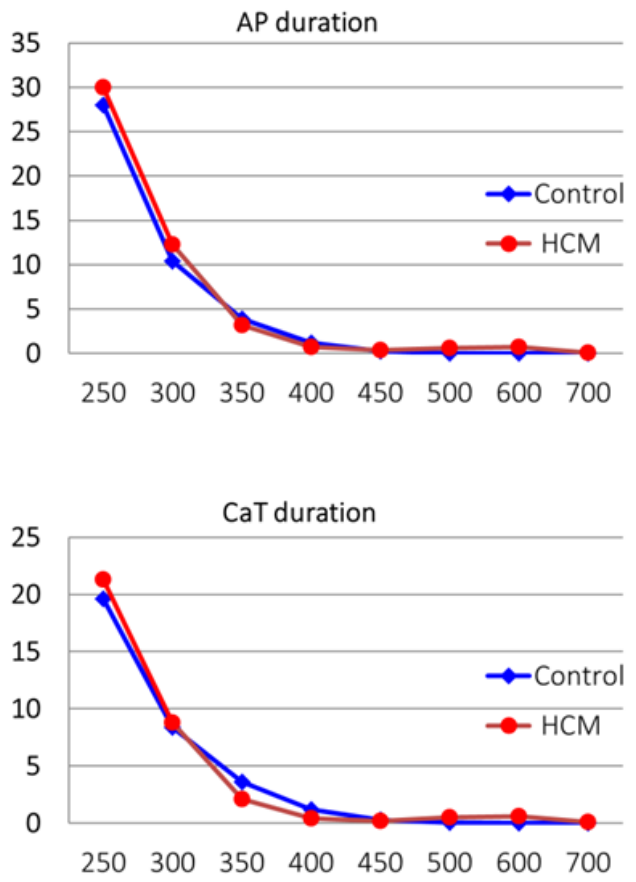

Pacing cycle length (ms)

Figure 1: Percentage of models displaying alternans for Control and HCM, for action potential duration and amplitude, and $\mathrm{Ca}^{2+}$ transient duration and amplitude at pacing cycle length from $250 \mathrm{~ms}$ to $700 \mathrm{~ms}$. 


\subsection{Comparison of alternans}

The percentage of models displaying alternans in control and HCM populations for different pacing lengths is represented on Figure 1.

For high cycle lengths ( $400 \mathrm{~ms}$ to $700 \mathrm{~ms}$ ), both groups show few APA alternans, with controls showing strictly no APA alternans at these frequencies.

For low cycle lengths (350ms to $250 \mathrm{~ms}$ ), HCM models show significantly more APA alternans than controls (250ms: $33 \%$ for HCM, $19 \%$ for Control, $300 \mathrm{~ms}: 13.4 \%$ for HCM, $2.6 \%$ for Control, 350ms: $3 \%$ for HCM, $0.1 \%$ for Control, all $\mathrm{p}<0.001)$. In addition, the magnitude of APA alternans (measured as the absolute difference between the two consecutive beats) is larger for HCM models compared to controls $(6.3 \pm 3.5 \mathrm{mV}$ vs $2.6 \pm 2.2 \mathrm{mV}$, $\mathrm{p}<0.001$ ), in agreement with previous studies [9].

For APD90, CaTA and CaT90 alternans, the HCM and control groups do not differ significantly in terms of percentage of models displaying alternans.

\subsection{Magnitude of alternans}

In addition to the number of models exhibiting alternans, we computed the magnitude of these alternans for both groups, measured as the absolute difference between the two consecutive beats. HCM models showed significantly higher magnitude of APA alternans compared to controls $(250 \mathrm{~ms}: 7.58 \pm 3.5 \mathrm{mV}$ vs $4.01 \pm$
$3.38 \mathrm{mV}, 300 \mathrm{~ms}: 6.32 \pm 3.52 \mathrm{mV}$ vs $2.61 \pm 2.26 \mathrm{mV}$, $350 \mathrm{~ms}: 4.59 \pm 2.63 \mathrm{mV}$ vs $1.27 \pm 0.26 \mathrm{mV}$, all $\mathrm{p}<0.001$, Figure 2, Panel A). In addition, HCM showed higher magnitude of APD alternans compared to controls (250ms: $21.61 \pm 8.29 \mathrm{~ms}$ vs $11.89 \pm 5.72 \mathrm{~ms}, 300 \mathrm{~ms}: 21.01$ $\pm 11.29 \mathrm{~ms}$ vs $9.81 \pm 3.79 \mathrm{~ms}, 350 \mathrm{~ms}: 18.69 \pm 9.90 \mathrm{~ms}$ vs $8.37 \pm 2.41 \mathrm{~ms}, 400 \mathrm{~ms}: 15.68 \pm 8.18 \mathrm{~ms}$ vs $7.66 \pm 2.01 \mathrm{~ms}$, $450 \mathrm{~ms}: 13.56 \pm 8.89 \mathrm{~ms}$ vs $6.61 \pm 1.32 \mathrm{~ms}$ all $\mathrm{p}<0.001$, all $\mathrm{p}<0.001$, Figure 2, Panel B).

\section{Discussion and conclusions}

In this work, we investigated the presence of alternans at the cellular level in HCM compared to controls using a population of models approach. HCM models displayed significantly more AP amplitude alternans than controls at low cycle lengths $(250-350 \mathrm{~ms})$, but no significant differences were observed at pacing cycle lengths between $400 \mathrm{~ms}$ and $1000 \mathrm{~ms}$. HCM and Control models also exhibited similar number of alternans in AP duration, $\mathrm{CaT}$ duration or $\mathrm{CaT}$ amplitude alternans at all frequencies. Despite low numbers, the magnitude of alternans in HCM models was significantly higher than in Controls in terms of action potential amplitude and duration.

A limitation of this approach is the threshold set for the computation of alternans ( $1 \%$ difference) capturing very subtle changes between two consecutive beats, suggesting that the alternans observed are not very marked. These
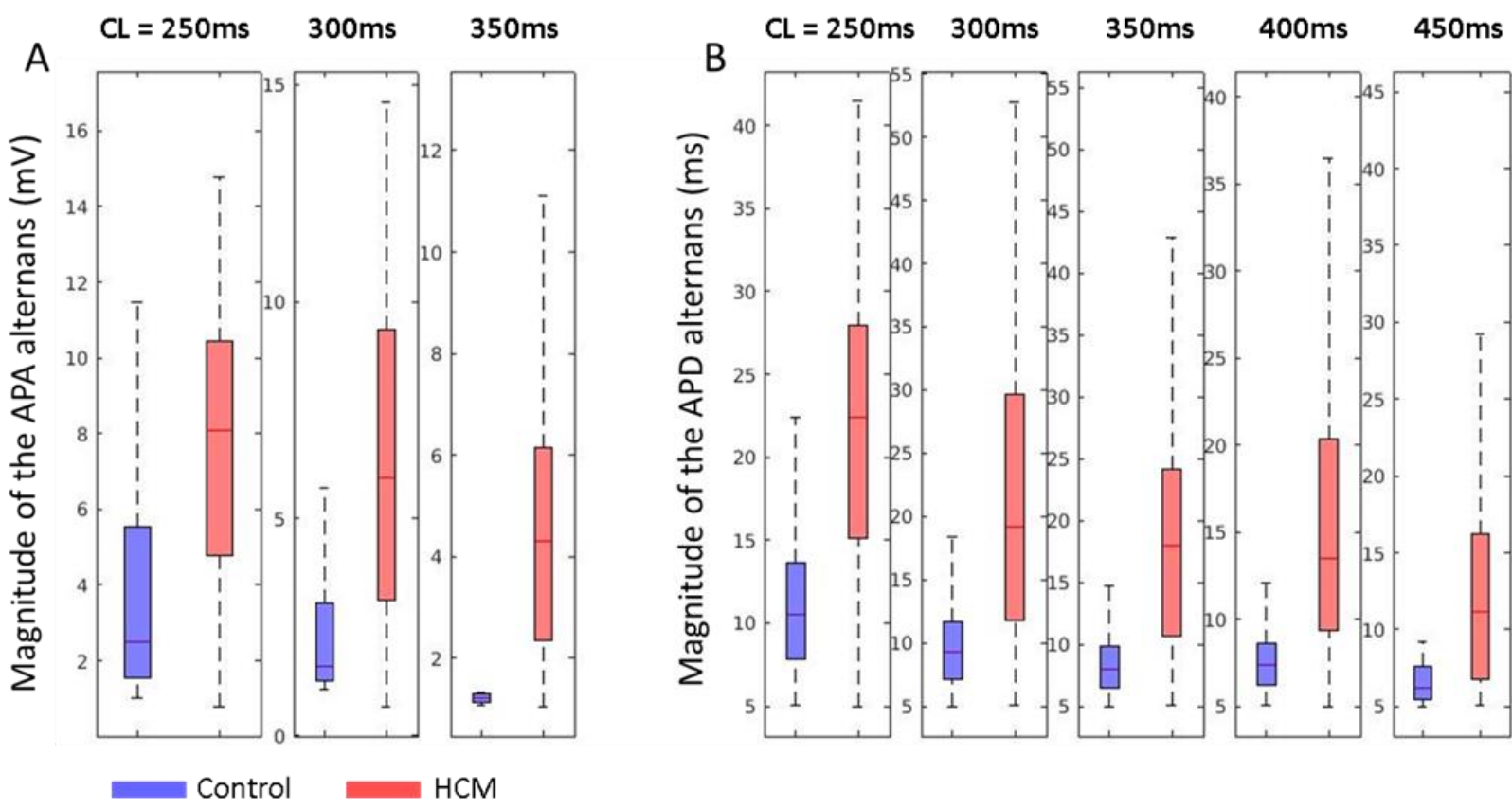

Figure 2: Boxplot comparing (A) the magnitude of AP amplitude alternans $(\mathrm{mV})$ in Control and HCM populations at 250, 300 and 350ms pacing length, (B) the magnitude of AP duration alternans (ms) in Control and HCM populations at $250,300,350,400$ and $450 \mathrm{~ms}$ pacing length 
results therefore do not strongly suggest that HCM cardiomyocytes are more prone to alternans in action potential biomarkers compared to Controls. This may suggest that TWA on the ECG, observed in HCM and proposed as potential tool for risk stratification [3], may be due to other dynamics than single cell abnormalities. Alteration of the tissue structure leading to heterogeneities and conduction delays may rather be the cause of TWA. Indeed, increased dispersion-ofrefractoriness in heterogeneous regions of the myocardium may lead to areas where recovery times are prolonged. Increased recovery may lead to partial conduction blocks and facilitate re-entry [14] [15]. Computer simulations have shown that refractoriness heterogeneities could lead to an alternating partial depolarization and result in repolarization alternans on the ECG [16]. More specifically in HCM, structural abnormalities like fibre disarray or fibrosis, which may create regional conduction abnormalities could in turn lead to an enhanced dispersion of refractoriness and promote the occurrence of pro-arrhythmic TWA as suggested in [17]. Further simulation studies could potentially investigate the influence of these abnormalities on the occurrence of alternating behaviours on the ECG.

\section{Acknowledgements}

AL is supported by a BHF CRE grant. AM and BR are supported by BR's Wellcome Trust Senior Research Fellowship in Basic Biomedical Sciences.

\section{References}

[1] N. Kuroda, Y. Ohnishi, A. Yoshida, A. Kimura, and M. Yokoyama, "Clinical Significance of T-Wave Alternans in Hypertrophic Cardiomyopathy," Circ. J., vol. 66, no. 5, pp. 457-462, 2002.

[2] Y. Momiyama et al., "Exercise-induced T-wave alternans as a marker of high risk in patients with hypertrophic cardiomyopathy," Jpn. Circ. J., vol. 61, no. 8, pp. 650656, Aug. 1997.

[3] M. de O. Antunes et al., "Exercise-induced quantitative microvolt T-wave alternans in hypertrophic cardiomyopathy," J. Electrocardiol., vol. 50, no. 2, pp. 184-190, Mar. 2017.

[4] R. L. Verrier, K. Kumar, and B. D. Nearing, "Basis for Sudden Cardiac Death Prediction by T-Wave Alternans from an Integrative Physiology Perspective," Heart Rhythm Off. J. Heart Rhythm Soc., vol. 6, no. 3, pp. 416422, Mar. 2009.

[5] M. J. Cutler and D. S. Rosenbaum, "Explaining the clinical manifestations of $\mathrm{T}$ wave alternans in patients at risk for sudden cardiac death," Heart Rhythm, vol. 6, no. 3 Suppl, pp. S22-28, Mar. 2009.

[6] R. Coppini et al., "Late sodium current inhibition reverses electromechanical dysfunction in human hypertrophic cardiomyopathy," Circulation, vol. 127, no. 5, pp. 575-
584, Feb. 2013.

[7] E. Passini et al., "Mechanisms of pro-arrhythmic abnormalities in ventricular repolarisation and antiarrhythmic therapies in human hypertrophic cardiomyopathy," J. Mol. Cell. Cardiol., vol. 96, pp. 7281, Jul. 2016.

[8] J. D. Bayer, S. M. Narayan, G. G. Lalani, and N. A. Trayanova, "Rate-dependent action potential alternans in human heart failure implicates abnormal intracellular calcium handling," Heart Rhythm Off. J. Heart Rhythm Soc., vol. 7, no. 8, pp. 1093-1101, Aug. 2010.

[9] S. M. Narayan, J. D. Bayer, G. Lalani, and N. A. Trayanova, "Action potential dynamics explain arrhythmic vulnerability in human heart failure: a clinical and modeling study implicating abnormal calcium handling," J. Am. Coll. Cardiol., vol. 52, no. 22, pp. 1782-1792, Nov. 2008.

[10] T. O’Hara, L. Virág, A. Varró, and Y. Rudy, "Simulation of the undiseased human cardiac ventricular action potential: model formulation and experimental validation," PLoS Comput. Biol., vol. 7, no. 5, p. e1002061, May 2011.

[11] G. R. Mirams et al., "Chaste: An Open Source C++ Library for Computational Physiology and Biology," PLOS Comput. Biol., vol. 9, no. 3, p. e1002970, Mar. 2013.

[12] X. Zhou et al., "In Vivo and In Silico Investigation into Mechanisms of Frequency Dependence of Repolarization Alternans in Human Ventricular Cardiomyocytes," Circ. Res., p. CIRCRESAHA.115.307836, Nov. 2015.

[13] F. Hua and R. F. Gilmour, "Contribution of IKr to ratedependent action potential dynamics in canine endocardium," Circ. Res., vol. 94, no. 6, pp. 810-819, Apr. 2004.

[14] C. S. Kuo, J. P. Amlie, K. Munakata, C. P. Reddy, and B. Surawicz, "Dispersion of monophasic action potential durations and activation times during atrial pacing, ventricular pacing, and ventricular premature stimulation in canine ventricles," Cardiovasc. Res., vol. 17, no. 3, pp. 152-161, Mar. 1983.

[15] A. A. Armoundas, G. F. Tomaselli, and H. D. Esperer, "Pathophysiological basis and clinical application of Twave alternans," J. Am. Coll. Cardiol., vol. 40, no. 2, pp. 207-217, Jul. 2002.

[16] J. M. Smith and R. J. Cohen, "Simple finite-element model accounts for wide range of cardiac dysrhythmias," Proc. Natl. Acad. Sci. U. S. A., vol. 81, no. 1, pp. 233-237, Jan. 1984.

[17] Y. Kon-No et al., "Microvolt T wave alternans in human cardiac hypertrophy: electrical instability and abnormal myocardial arrangement," J. Cardiovasc. Electrophysiol., vol. 12 , no. 7, pp. 759-763, Jul. 2001.

Address for correspondence.

Name. Aurore Lyon

Full postal address. Department of Computer Science, Parks

Road, Oxford, OX1 3QD

E-mail address. aurore.lyon@cs.ox.ac.uk 